\title{
How information geometry is encoded in bulk geometry
}

\author{
Asato Tsuchiya* \\ Department of Physics, Shizuoka University, 836 Ohya, Suruga-ku, Shizuoka 422-8529, Japan \\ E-mail: tsuchiva.asatodshizuoka.ac. ip
}

We study how information geometry is encoded in bulk geometry. We consider a quantum information metric that measures the distance between the ground states of a CFT and a theory obtained by perturbing the CFT. We find a universal formula that represents the quantum information metric in terms of back reaction to the AdS bulk geometry.

Corfu Summer Institute 2019 "School and Workshops on Elementary Particle Physics and Gravity" (CORFU2019)

31 August - 25 September 2019

Corf 湛, Greece

${ }^{*}$ Speaker. 


\section{Introduction}

Emergent geometry seems to play a crucial role in constructing quantum theory of gravity. It is, indeed, seen in various contexts such as matrix models and the AdS/CFT correspondence or the gauge/gravity correspondence [1-3]. In the AdS/CFT correspondence, the bulk direction on the gravity side emerges as the scale of renormalization group on the field theory side [2-5]. This observation raises a problem: can one reconstruct full bulk geometry from field theory? The RyuTakayanagi formula [6] provides insight into this problem. It expresses entanglement entropy of a region in space on which a field theory is defined by the area of a minimal surface in the bulk whose boundary coincides with that of the region. Thus, it gives a relation between quantum information theory and gravity.

In this talk, in order to gain further insights into this problem, we consider quantum information metric in quantum information theory other than entanglement entropy. We consider a CFT and a theory that is obtained by perturbing the CFT by an operator, and calculate a quantum information metric that measures the distance between the ground states of these two theories. We find a formula that represents the quantum information metric in terms of back reaction to the AdS bulk geometry, which is determined by dynamics of gravity. This formula is universal in the sense that it holds for each case in which the perturbation is given by a scalar, vector or tensor operator. The geometrical quantity associated with the quantum information metric is local in the bulk direction, while the minimal surface associated with entanglement entropy is not. This talk is based on [7]. Information metrics have been investigated in the context of the AdS/CFT correspondence in $[8-16]$.

\section{Quantum information metric in field theory}

We consider a field theory defined on $\mathbb{R}^{d}$ with the coordinates $(\tau, \vec{x})$, where $\tau$ is the Euclidean time and $\vec{x}$ are $(d-1)$-dimensional space coordinates. We consider two theories, the theory 1 and the theory 2 that are defined on the same Hilbert space and have the lagrangians, $\mathscr{L}_{1}$ and $\mathscr{L}_{2}$, respectively. The ground states of these two theories are denoted by $\left|\Omega_{1}\right\rangle$ and $\left|\Omega_{2}\right\rangle$, respectively. Then, the inner product between the ground states is represented in terms of a path integral as

$$
\left\langle\Omega_{2} \mid \Omega_{1}\right\rangle=\frac{1}{\left(Z_{1} Z_{2}\right)^{1 / 2}} \int \mathscr{D} \psi \exp \left[-\int d^{d-1} x\left(\int_{-\infty}^{0} d \tau \mathscr{L}_{1}+\int_{0}^{\infty} d \tau \mathscr{L}_{2}\right)\right]
$$

where $Z_{1}$ and $Z_{2}$ are the partition functions of the theory 1 and the theory 2 , respectively.

By denoting the difference of the two lagrangians by $\delta \mathscr{L}$, that is, $\mathscr{L}_{2}=\mathscr{L}_{1}+\delta \mathscr{L}$, we rewrite the inner product (L.]) in terms of $\delta \mathscr{L}$ as

$$
\left\langle\Omega_{2} \mid \Omega_{1}\right\rangle=\frac{\left\langle\exp \left[-\int_{0}^{\infty} d \tau \int d^{d-1} x \delta \mathscr{L}\right]\right\rangle_{1}}{\left\langle\exp \left[-\int_{-\infty}^{\infty} d \tau \int d^{d-1} x \delta \mathscr{L}\right]\right\rangle_{1}^{1 / 2}},
$$

where \langle\rangle$_{1}$ stands for the vacuum expectation value taken with respect to the theory 1 :

$$
\langle\mathscr{O}\rangle_{1}=\frac{1}{Z_{1}} \int \mathscr{D} \psi \mathscr{O} e^{-S_{1}}=\left\langle\Omega_{1}|\mathscr{O}| \Omega_{1}\right\rangle
$$


We expand ([2.) in terms of $\delta \mathscr{L}$ up to $\mathscr{O}\left(\delta \mathscr{L}^{2}\right)$ and define the quantum information metric $\mathscr{G}$, which is known as Fisher's metric and measures the distance between the ground states of the two theories, by

$$
\mathscr{G}=\frac{1}{T}\left(1-\left\langle\Omega_{2} \mid \Omega_{1}\right\rangle\right),
$$

where $T$ is the volume of time direction. Then, some algebra leads to [9]

$$
\mathscr{G}=\frac{1}{2 T} \int_{0}^{\infty} d \tau \int_{-\infty}^{0} d \tau^{\prime} \int d^{d-1} x \int d^{d-1} x^{\prime}\left\langle\delta \mathscr{L}(\tau, \vec{x}) \delta \mathscr{L}\left(\tau^{\prime}, \vec{x}^{\prime}\right)\right\rangle_{1}
$$

where

$$
\langle\delta \mathscr{L}(\tau, \vec{x})\rangle_{1}=0
$$

and the time reversal symmetry

$$
\left\langle\delta \mathscr{L}(\tau, \vec{x}) \delta \mathscr{L}\left(\tau^{\prime}, \vec{x}\right)\right\rangle_{1}=\left\langle\delta \mathscr{L}(-\tau, \vec{x}) \delta \mathscr{L}\left(-\tau^{\prime}, \vec{x}\right)\right\rangle_{1}
$$

are assumed.

Let us consider a case in which the theory 1 is a CFT and $\delta \mathscr{L}(\tau, \vec{x})=\phi(\vec{x}) \mathscr{O}(\tau, \vec{x})$ with $\mathscr{O}(\tau, \vec{x})$ being a scalar primary operator with the conformal dimension $\Delta$ in the CFT. Namely, the theory 2 is obtained by perturbing the CFT by the scalar primary operator. Note that the source $\phi(\vec{x})$ is independent of $\tau$. In what follows, the quantities in the theory 1 are labeled 'CFT' instead of ' 1 ', while those in the theory 2 have no labels. For instance,

$$
\mathscr{L}=\mathscr{L}_{C F T}+\phi(\vec{x}) \mathscr{O}(\tau, \vec{x})
$$

and so on.

The one-point function of the primary operator $\mathscr{O}$ vanishes, which implies that (2.6) is satisfied. The two-point function of the primary operator $\mathscr{O}$ takes the form

$$
\left\langle\mathscr{O}(\tau, \vec{x}) \mathscr{O}\left(\tau^{\prime}, \vec{x}^{\prime}\right)\right\rangle_{C F T}=\frac{C_{\Delta}}{\left(\varepsilon^{2}+\left(\tau-\tau^{\prime}\right)^{2}+\left(\vec{x}-\vec{x}^{\prime}\right)^{2}\right)^{\Delta}},
$$

where $C_{\Delta}$ is a normalization constant and a UV cutoff $\varepsilon$ has been introduced. We see from (2.) that $(\mathbb{2 . 7})$ is satisfied.

The information metric (2.5]) reads

$$
\mathscr{G}=\frac{1}{8} \int_{-\infty}^{\infty} d s \int d^{d-1} x \int d^{d-1} x^{\prime} \frac{C_{\Delta} \phi(\vec{x}) \phi\left(\vec{x}^{\prime}\right)}{\left(\varepsilon^{2}+s^{2}+\left(\vec{x}-\vec{x}^{\prime}\right)^{2}\right)^{\Delta}} .
$$

\section{Quantum information metric as on-shell action}

Suppose that the CFT has a gravity dual defined on $A d S_{d+1}$. Throughout this talk, we consider a situation in which the classical approximation is valid on the gravity side. Because the quantum information metric takes the form of the generating functional for the two-point functions, it can be represented by the on-shell action for the bulk field $\Phi$ dual to $\mathscr{O}$. 
Here we introduce the following notations: $x^{\mu}=\left(x^{0}, x^{i}\right)=(\tau, \vec{x})$, where $\mu=0, \ldots, d-1$ and $i=1, \ldots, d-1$, and $z^{M}=\left(z, x^{\mu}\right)$. We use the metric of $A d S_{d+1}$ in the Poincare coordinates, which takes the form

$$
d s^{2}=G_{M N} d z^{M} d z^{N}=\frac{1}{z^{2}}\left(d z^{2}+d x^{\mu} d x^{\mu}\right) .
$$

We define a boundary hypersurface in $A d S_{d+1}$ by $z=\varepsilon$, where $\varepsilon$ was introduced in (2.9) as a UV cutoff. The CFT is viewed as defined on the boundary.

The action for $\Phi$ on the gravity side is

$$
S_{M}=\frac{1}{2} \int d^{d+1} x \sqrt{G}\left(G^{M N} \partial_{M} \Phi \partial_{N} \Phi+\Delta(\Delta-d) \Phi^{2}\right),
$$

where we have presented only the quadratic terms in $\Phi$, which are needed in the following.

The equation of motion for $\Phi$ is derived from (B.2) as

$$
-\frac{1}{\sqrt{G}} \partial_{M}\left(\sqrt{G} G^{M N} \partial_{N} \Phi\right)+\Delta(\Delta-d) \Phi=0 .
$$

The boundary condition for $\Phi$ is given by

$$
\Phi(z=\varepsilon, \tau, \vec{x})=\varepsilon^{d-\Delta} \phi(\vec{x}) .
$$

The solution to (B.3]) satisfying the boundary condition $\Phi(z=\varepsilon, \tau, \vec{x})=\varepsilon^{d-\Delta} \phi(\vec{x})$ is [3]

$$
\Phi(z, x)=\int d^{d} x^{\prime} K\left(z, x-x^{\prime}\right) \phi\left(\vec{x}^{\prime}\right)
$$

where $K$ is the so-called boundary to bulk propagator:

$$
K(z, x)=\frac{\alpha_{\Delta} z^{\Delta}}{\left(z^{2}+x^{2}\right)^{\Delta}} \text { with } \alpha_{\Delta}=\frac{\Gamma(\Delta)}{\pi^{\frac{d}{2}} \Gamma\left(\Delta-\frac{d}{2}\right)} .
$$

Note that $\Phi(z, x)$ is independent of $\tau$.

By substituting (B.5) into (B.2) and using (B.3), we evaluate the on-shell action for $\Phi$ as follows:

$$
\begin{aligned}
S_{\text {on-shell }} & =\frac{1}{2} \int d^{d+1} x \partial_{M}\left(\sqrt{G} G^{M N} \Phi \partial_{N} \Phi\right) \\
& =-\frac{1}{2} \int_{z=\varepsilon} d^{d} x \varepsilon^{-d+1} \Phi \partial_{z} \Phi \\
& =-\Delta \alpha_{\Delta} \int_{0}^{\infty} d \tau \int_{-\infty}^{\infty} d s \int d^{d-1} x d^{d-1} x^{\prime} \frac{\phi(\vec{x}) \phi\left(\vec{x}^{\prime}\right)}{\left(\varepsilon^{2}+s^{2}+\left(\vec{x}-\vec{x}^{\prime}\right)^{2}\right)^{\Delta}}
\end{aligned}
$$

This would be the generating functional of $\phi(\vec{x})$ for two-point functions of $\mathscr{O}$ so that we obtain

$$
C_{\Delta}=\Delta \alpha_{\Delta}
$$

Then, by comparing (B.]) with ([2.10), we find

$$
S_{\text {on-shell }}=-4 T \mathscr{G} .
$$




\section{Back reaction to the AdS geometry}

The theory (․8) obtained by perturbing the CFT by the primary operator would have a gravity dual where the geometry gains a back reaction to the AdS geometry, namely deviates from the AdS geometry. We evaluate the back reaction up to $\mathscr{O}\left(\phi^{2}\right)$ in the following.

We parametrize the metric with the back reaction as

$$
d s^{2}=G_{M N} d z^{M} d z^{N}=\frac{1}{z^{2}}\left(d z^{2}+g_{\mu v}(z, x) d x^{\mu} d x^{v}\right),
$$

with

$$
g_{\mu v}(z, x)=\delta_{\mu v}+h_{\mu v}(z, x)
$$

where $h_{\mu v}$ represent the back reaction to the AdS geometry and start with $\mathscr{O}\left(\phi^{2}\right)$ contribution, which we will focus on.

The gravity action on the gravity side is given by

$$
S_{G}=\frac{1}{16 \pi G_{N}}\left[\int d^{d+1} x \sqrt{G}(-R[G]-d(d-1))-\int_{z=\varepsilon} d^{d} x \sqrt{\gamma}(2 K-2(d-1))\right],
$$

where $\gamma$ is the induced metric on the boundary, and $K$ is the trace of the extrinsic curvature.

The Einstein equation is derived from (4.3]) and (B.2) as

$$
\begin{aligned}
& \operatorname{Tr} g^{-1} g^{\prime \prime}-\frac{1}{z} \operatorname{Tr} g^{-1} g^{\prime}-\frac{1}{2} \operatorname{Tr} g^{-1} g^{\prime} g^{-1} g^{\prime}=-16 \pi G_{N} \mathscr{T}_{z z}, \\
& \nabla_{\mu} \operatorname{Tr} g^{-1} g^{\prime}-\nabla^{\lambda} g_{\lambda \mu}^{\prime}=-16 \pi G_{N} \mathscr{T}_{z \mu}, \\
& g_{\mu \nu}^{\prime \prime}-g_{\mu \lambda}^{\prime} g^{\lambda \sigma} g_{\sigma v}^{\prime}+\frac{1}{2} \operatorname{Tr}\left(g^{-1} g^{\prime}\right) g_{\mu \nu}^{\prime}-(d-1) \frac{1}{z} g_{\mu v}^{\prime}-\frac{1}{z} \operatorname{Tr}\left(g^{-1} g^{\prime}\right) g_{\mu v}-2 R(g)_{\mu \nu} \\
& =-16 \pi G_{N} \mathscr{T}_{\mu \nu}
\end{aligned}
$$

with

$$
\begin{aligned}
& \mathscr{T}_{z z}=\partial_{z} \Phi \partial_{z} \Phi+\frac{\Delta(\Delta-d)}{d-1} \frac{1}{z^{2}} \Phi^{2}, \\
& \mathscr{T}_{z \mu}=\partial_{z} \Phi \partial_{\mu} \Phi, \\
& \mathscr{T}_{\mu \nu}=\partial_{\mu} \Phi \partial_{\nu} \Phi+\frac{\Delta(\Delta-d)}{d-1} \frac{1}{z^{2}} g_{\mu \nu} \Phi^{2},
\end{aligned}
$$

where the prime stands for the derivative with respect to $z$.

We expand the left hand sides up to $\mathscr{O}\left(h_{\mu v}\right)$ in order to evaluate $h_{\mu v}$ up to $\mathscr{O}\left(\phi^{2}\right)$, since the righthand sides of (4.4), (4.5) and (4.6) are $\mathscr{O}\left(\phi^{2}\right)$. Here, $h_{\mu \nu}$ is independent of $\tau$, because so is $\Phi$, and we ignore total derivative terms with respect to $\vec{x}$, which will be justified shortly. Then, (4.4) reduces to

$$
\operatorname{Tr} h^{\prime \prime}-\frac{1}{z} \operatorname{Tr} h^{\prime}=-16 \pi G_{N}\left(\partial_{z} \Phi \partial_{z} \Phi+\frac{\Delta(\Delta-d)}{d-1} \frac{1}{z^{2}} \Phi^{2}\right)
$$


while (4.6) reduces to

$$
h_{\mu \nu}^{\prime \prime}-(d-1) \frac{1}{z} h_{\mu \nu}^{\prime}-\frac{1}{z} \operatorname{Tr} h^{\prime} \delta_{\mu v}=-16 \pi G_{N}\left(\partial_{\mu} \Phi \partial_{\nu} \Phi+\frac{\Delta(\Delta-d)}{d-1} \frac{1}{z^{2}} \delta_{\mu v} \Phi^{2}\right) .
$$

Taking the trace of (4.9) yields

$$
\operatorname{Tr} h^{\prime \prime}-(2 d-1) \frac{1}{z} \operatorname{Tr} h^{\prime}=-16 \pi G_{N}\left(\partial_{\mu} \Phi \partial_{\mu} \Phi+\frac{\Delta(\Delta-d)}{d-1} \frac{d}{z^{2}} \Phi^{2}\right) .
$$

The 00 component of (4.9) reads

$$
h_{00}^{\prime \prime}-(d-1) \frac{1}{z} h_{00}^{\prime}-\frac{1}{z} \operatorname{Tr} h^{\prime}=-16 \pi G_{N} \frac{\Delta(\Delta-d)}{d-1} \frac{1}{z^{2}} \Phi^{2} .
$$

By taking a linear combination of (4.8), (4.10) and (4.1) and using (B.3), we obtain

$$
\operatorname{tr} h^{\prime \prime}-\frac{d-1}{z} \operatorname{tr} h^{\prime}=-8 \pi G_{N}\left\{\partial_{z}\left(\Phi \partial_{z} \Phi\right)-\frac{d-1}{z} \Phi \partial_{z} \Phi\right\},
$$

where $\operatorname{tr} A=A_{i i}=\operatorname{Tr} A-A_{00}$. Integrating (4.12) leads to

$$
\operatorname{tr} h^{\prime}=-8 \pi G_{N} \Phi \partial_{z} \Phi
$$

where the boundary condition $\lim _{z \rightarrow \infty} h_{\mu v}=0$ has been used.

By using the third equality in (B.7) and (4.13), we obtain

$$
S_{\text {on-shell }}=\frac{1}{16 \pi G_{N}} \int_{z=\varepsilon} d^{d} x \varepsilon^{-d+1} \operatorname{tr} h^{\prime} .
$$

Here ignoring the total derivative terms in deriving (4.53) is justified.

By comparing (B. 3 ) and (4.5), we find a formula

$$
\mathscr{G}=-\frac{1}{64 \pi G_{N}} \int_{z=\varepsilon} d^{d-1} x \varepsilon^{-d+1} \operatorname{tr} h^{\prime}
$$

This formula represents the information metric in field theory in terms of buck reaction to the AdS bulk geometry. The righthand side of (4.15) is interpreted geometrically as follows.

We consider a hypersurface specified by $z=\varepsilon$ and $\tau=$ const.. The induced metric on the hypersurface in the static gauge is given by

$$
k_{i j}=\frac{1}{z^{2}} g_{i j} .
$$

The volume of the hypersurface is evaluated up to $\mathscr{O}\left(h_{i j}\right)$ as

$$
\begin{aligned}
v & =\int_{z=\varepsilon} d^{d-1} x \sqrt{k} \\
& =\int_{z=\varepsilon} d^{d-1} x z^{-d+1}\left(1+\frac{1}{2} \operatorname{tr} h\right) .
\end{aligned}
$$


We subtract the volume of the hypersurface in the AdS case where $h_{i j}=0$ and denote the difference by $\delta v$ :

$$
\delta v=\frac{1}{2} \int_{z=\varepsilon} d^{d-1} x z^{-d+1} \operatorname{trh}
$$

By taking the derivative with respect to $z$, we obtain

$$
\delta v^{\prime}=\frac{1}{2} \int_{z=\varepsilon} d^{d-1} x\left((-d+1) z^{-d} \operatorname{tr} h+z^{-d+1} \operatorname{tr} h^{\prime}\right) .
$$

While the first term in (4.19) represents the canonical scaling of the volume, the second term represents a nontrivial scaling of the volume and is proportional to the righthand side of (4. (4) ). Thus, (4.5) is rewritten as

$$
\mathscr{G}=-\frac{1}{32 \pi G_{N}} \delta v_{\text {nontrivial }}^{\prime}
$$

with

$$
\delta v_{\text {nontrivial }}^{\prime}=\frac{1}{2} \int_{z=\varepsilon} d^{d-1} x z^{-d+1} \operatorname{tr} h^{\prime} .
$$

The formula (4.15) holds also for the case in which the perturbation to the CFT is given by a vector or tensor operator.

\section{Conclusion and discussion}

In this talk, we studied how information geometry is encoded in bulk geometry. We considered a quantum information metric that measures the distance between the ground states of a CFT and a theory obtained by perturbing the CFT. We represented the information metric in terms of the back reaction that the bulk geometry gains due to the perturbation. We found the formula (4.20) that represents the information metric in terms of back reaction to the AdS bulk geometry. The geometrical quantity related to the information metric in the formula is local in the bulk direction. The formula is universal in the sense that it holds for each case of a scalar, vector or tensor perturbation. It associates information geometry with dynamics of gravity.

We associated the information metric with the volume of a hypersurface specified by $z=\varepsilon$ with $\varepsilon$ small and $\tau=$ const.. In order to reconstruct full bulk geometry from field theory, we should associate it with a hypersurface specified by $z=$ an arbitrary constant by using renormalization group. Furthermore, we need to find relationship between information geometry and bulk quantities local even in $\vec{x}$ directions. It should also be crucial to derive effects of strings and quantum gravity from information geometry to construct quantum theory of gravity.

\section{Acknowledgements}

The author would like to thank Kazushi Yamashiro for collaboration. The author was supported in part by Grant-in-Aid for Scientific Research (No. 18K03614) from Japan Society for the Promotion of Science. 


\section{References}

[1] J. M. Maldacena, Int. J. Theor. Phys. 38, 1113 (1999) [Adv. Theor. Math. Phys. 2, 231 (1998)] [hep-th/9711200].

[2] S. S. Gubser, I. R. Klebanov and A. M. Polyakov, Phys. Lett. B 428, 105 (1998) [hep-th/9802109].

[3] E. Witten, Adv. Theor. Math. Phys. 2, 253 (1998) [hep-th/9802150].

[4] L. Susskind and E. Witten, hep-th/9805114.

[5] J. de Boer, E. P. Verlinde and H. L. Verlinde, JHEP 0008, 003 (2000) [hep-th/9912012].

[6] S. Ryu and T. Takayanagi, Phys. Rev. Lett. 96, 181602 (2006) [hep-th/0603001].

[7] A. Tsuchiya and K. Yamashiro, [arXiv:2002.11365 [hep-th]].

[8] M. Nozaki, S. Ryu and T. Takayanagi, JHEP 1210, 193 (2012) [arXiv:1208.3469 [hep-th]].

[9] M. Miyaji, T. Numasawa, N. Shiba, T. Takayanagi and K. Watanabe, Phys. Rev. Lett. 115, no. 26, 261602 (2015) [arXiv:1507.07555 [hep-th]].

[10] N. Lashkari and M. Van Raamsdonk, JHEP 1604, 153 (2016) [arXiv:1508.00897 [hep-th]].

[11] D. Bak, Phys. Lett. B 756, 200 (2016) [arXiv:1512.04735 [hep-th]].

[12] A. Trivella, Class. Quant. Grav. 34, no. 10, 105003 (2017) [arXiv:1607.06519 [hep-th]].

[13] S. Aoki and S. Yokoyama, PTEP 2018, no. 3, 031 B01 (2018) [arXiv:1707.03982 [hep-th]].

[14] C. B. Chen, W. C. Gan, F. W. Shu and B. Xiong, Phys. Rev. D 98, no. 4, 046008 (2018) [arXiv:1804.08358 [hep-th]].

[15] A. May and E. Hijano, JHEP 1810, 036 (2018) [arXiv:1806.06077 [hep-th]].

[16] S. Karar, R. Mishra and S. Gangopadhyay, Phys. Rev. D 100, no. 2, 026006 (2019) [arXiv:1904.13090 [hep-th]].

[17] H. Liu and A. A. Tseytlin, Nucl. Phys. B 533, 88 (1998) [hep-th/9804083]. 